\title{
Restoration of a Traumatically Amputated Finger by a Traditional Glove-Type Prosthesis: A Case Report
}

\author{
Simarpreet Kaur, Mandeep Kaur, Anupama Verma, and Tarun Singh
}

\section{ABSTRACT}

Background: Each organ serves a specific function in the human body. Congenital absence or loss of even minor parts of it can pose extreme psychological trauma in addition to the functional impairment and esthetic issues encountered by the individual. Amputation of complete or a part of phalange of hand is frequently encountered either due to trauma or necrosis. Restoration of such defects become mandatory to improve general form and function combined with the esthetic requirements of the patient. It can either be done by microvascular reconstruction or prosthetic rehabilitation. The latter becomes the means of choice in cases where the former is either not possible, unavailable, unsuccessful, or unaffordable.

Case Report: A case report has been presented of a 60 years old patient with an old traumatic partially amputated index finger.

Treatment Plan: A custom-made glove-type prosthesis was fabricated using silicone elastomer.

Conclusion: A ring is provided for better esthetics and special adhesive is recommended for retention of the prosthesis.

Keywords: Finger Amputation, Glove Type Prosthesis, Rehabilitation,

Silicone Material.
Submitted : May 02, 2021

Published : May 28, 2021

ISSN: $2593-8339$

DOI: $10.24018 /$ ejmed.2021.3.3.853

Dr. Simarpreet Kaur *

BDS, Post Graduate Student, Department of Prosthodontics and Crown \& Bridge, Luxmi Bai Institute of Dental Sciences, Patiala, India.

(e-mail: simarmehra7869@gmail.com)

Dr. Mandeep Kaur

BDS, MDS, Apex Dental Care, Patiala, India.

(e-mail: dr.mandeepkaur@ymail.com)

Dr. Anupama Verma

BDS, Post Graduate Student, Department of Prosthodontics and Crown \& Bridge, Luxmi Bai Institute of Dental Sciences, Patiala, India.

(e-mail: anupamaverma2508@gmail.com) Dr. Tarun Singh

BDS, MDS, Consultant Oral and Maxillofacial Surgeon, JSM Multispecialty Dental Clinic, S.A.S. Nagar, India. (e-mail: tarunkashyap95@gmail.com)

*Corresponding Author

\section{INTRODUCTION}

Amputation of complete or a part of phalange of hand is a frequently encountered scenario. Allen's classification is frequently used to outline the level of fingertip amputations. Type 1 injuries are those involving only the pulp. Type 2 injuries consist of injury to the pulp and nail bed. Type 3 injuries include distal phalangeal fracture with associated pulp and nail loss. Type 4 injuries involve the lunula, distal phalanx, pulp and nail loss [1]. The main causes of finger amputation are infections and nerve injuries, congenital malformation, malignancy, traumatic injuries, such as frost bite, gun shot, burns and occupational accidents [2]. The incidence of traumatic amputation is higher in developing countries, like India, due to negligence in use of personal protective measures among agricultural and factory workers. Loss of either a complete digit or a part of it, in addition to functional inefficacy results in lack of esthetic appeal. This further can pose a serious detrimental effect on the psychological well-being of the individual. Therefore, restoration of certain defects become important. It can either be done by microvascular reconstruction or prosthetic rehabilitation. Prosthetics is an art and science of supplying artificial replacements for missing parts of human body [3]. It is the means opted for the restoration when microvascular reconstruction is either not possible, unavailable, unsuccessful, or unaffordable. Most common prosthetic methods to replace the loss of finger are Endoprosthesis, e.g., implants and Exoprosthesis, e.g., silicon prosthesis, acrylic prosthesis, prosthesis using attachments, and magnets. Creating a finger prosthesis that appears to have a realistic skin surface while achieving seamless visual integration with the surrounding tissue requires both artistic and technical expertise [4]. Passing through various materials, the acceptance rate has been much higher with an individually sculpted custom restoration using silicone elastomer [5], [6]. This study presents a case report of restoration of an indexfinger defect with a glove type silicone prosthesis and elaborates the method of retention for the same. 


\section{CASE REPORT}

A 60-year-old male patient visited the Department of Prosthodontics in Luxmi Bai Institute of Dental Sciences, Patiala with a chief complaint of partially missing index finger of his right-hand. History revealed that the patient lost it 5 years back due to an agricultural traumatic injury. The amputation was partial, involving the middle part of the proximal phalange of the index finger of right hand. On physical examination, there was no pain on palpation, and the surrounding skin showed zero inflammation or suppuration but appeared to be stretched (Fig. 1). The patient had no history of any preceding prosthesis in regard to it. The treatment options included implant-supported fixed prosthesis and conventional silicone removable prosthesis. As the patient was unwilling for any surgical procedure, fabrication of conventional silicone prosthesis was planned.

\section{A. Method of Fabrication: Impression Making}

A fine layer of petroleum jelly was applied on the patient's index finger stump and impression was made with the help of rubber base impression material (GcFlexceed Putty and Kit) which was poured with ADA type IV die stone (Kal Rock, Kala Bhai Karson Pvt.) to reduplicate the amputated finger and its associated structures (Fig. 2).
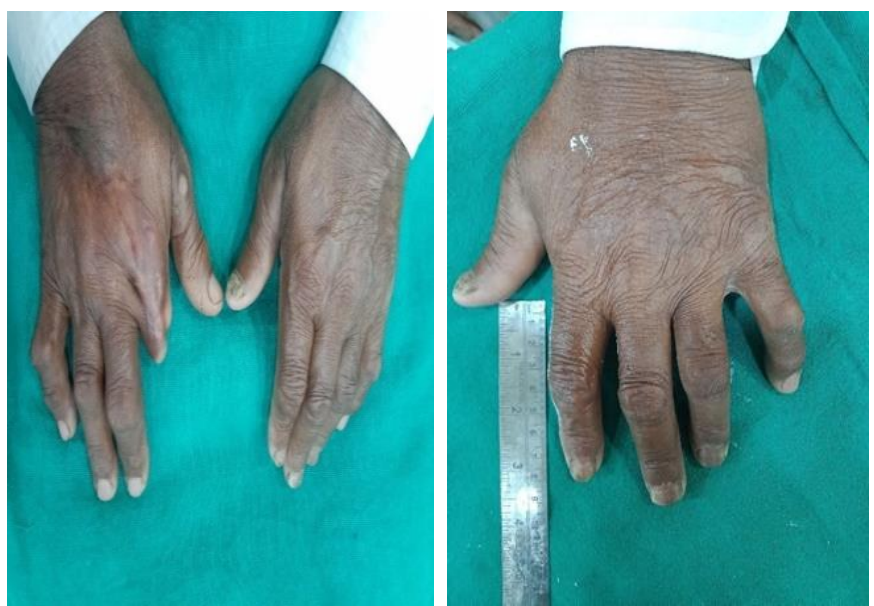

Fig. 1. Measurement of Index Finger of Contralateral Hand.

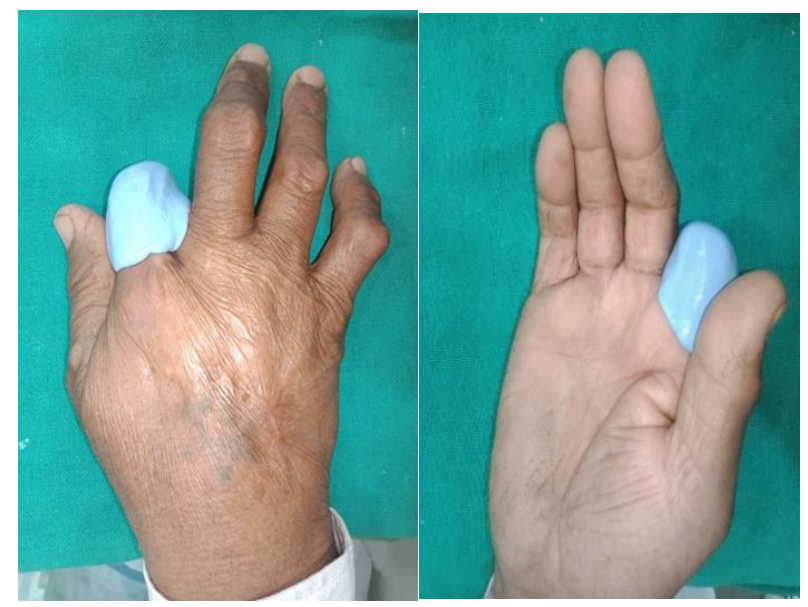

Fig. 2. Putty Impression of the Amputated Stump.
A fine layer of petroleum jelly was applied on the patient's index finger of left-hand prior making an impression. A disposable glass was selected according to the length of the index finger of the left hand. Impression of the same was made with irreversible hydrocolloid (Zelgan, 2002, DENTSPLY) impression material by asking the patient to place his finger in relaxed state in the selected disposable glass without touching its bottom part (Fig. 3). Modelling wax (Rolex modelling wax no. 2) was melted and poured into the impression to duplicate the anatomic details for the lost finger (Fig. 4).

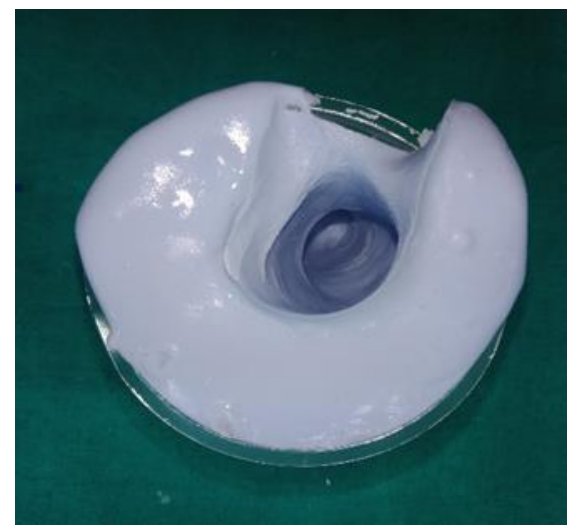

Fig. 3. Irreversible Hydrocolloid Impression of the Index Finger of Contralateral Hand.

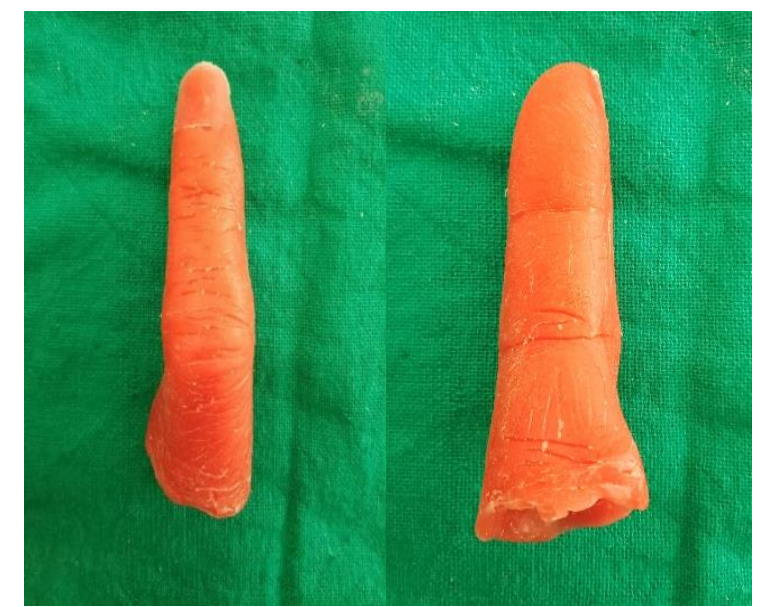

Fig. 4. Wax Pattern Modified to Resemble the Contours of the Amputated Finger.

\section{B. Wax Pattern Fabrication}

The wax pattern formed was placed over the duplicated finger stump and necessary modifications in thickness and anatomy were made. Surface characterizations (skin folds and wrinkles) were incorporated so as to reproduce the exact anatomy of the missing finger. This pattern was gauged and further modified keeping in mind the alignment, size, shape, and silhouette of the contralateral finger. Wax pattern trial was done to check the fit and alignment with respect to adjacent fingers (Fig. 5-7). 


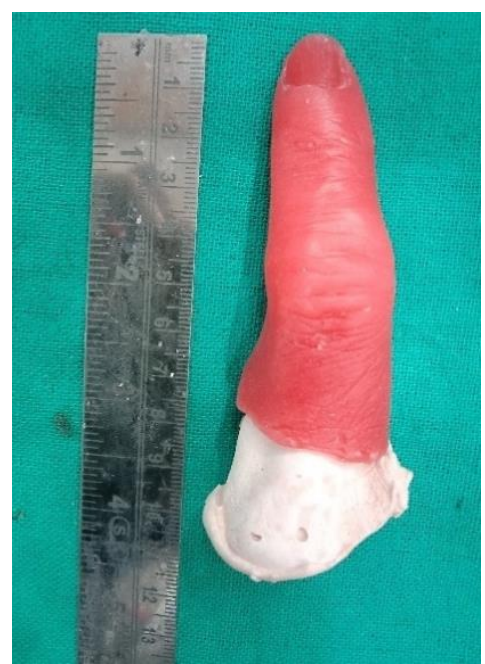

Fig. 5. Wax Pattern Adapted on Mould of the Stump.

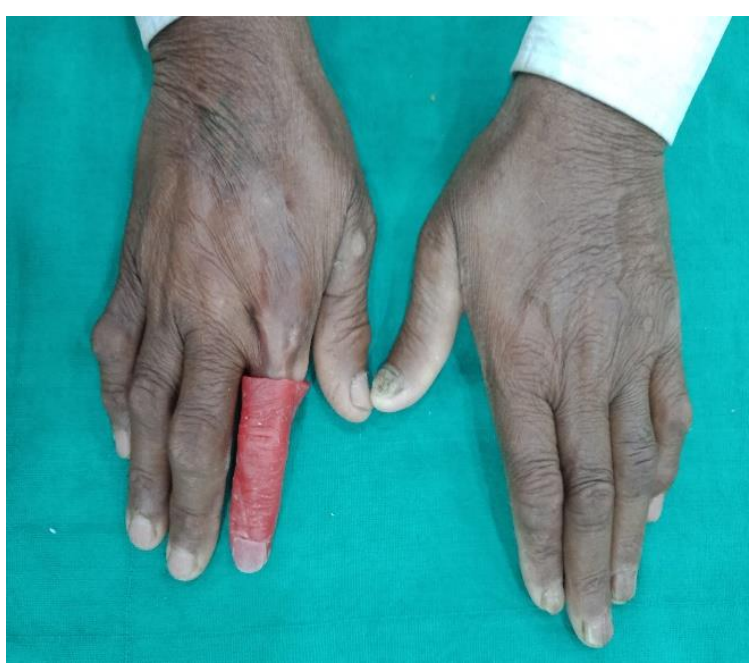

Fig. 6. Wax Pattern Trial.

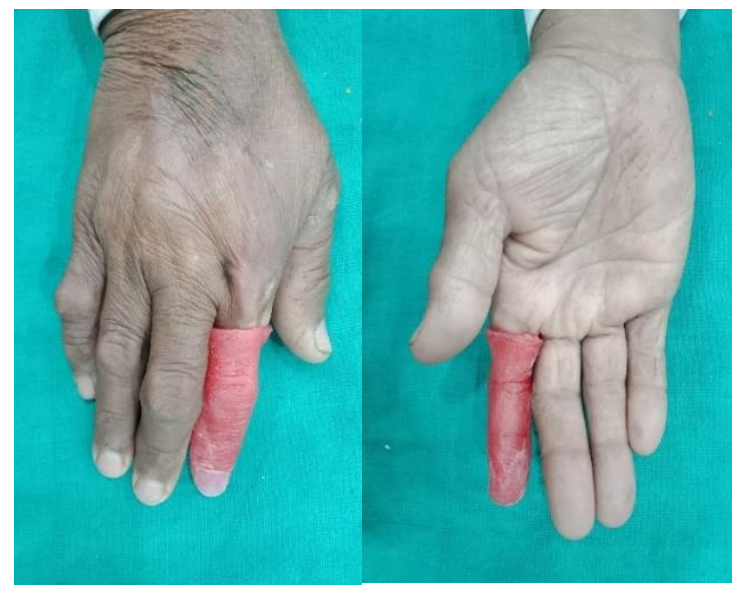

Fig. 7. Wax Pattern Trial.

The HTV maxillofacial silicone was mixed following manufacturer's instructions, avoiding any incorporation of air bubbles. Shade matching was carried out by adding an appropriate ratio of intrinsic colors (MP Sai biomed silicone prosthesis - a set of five pigments) available with silicone material under natural light. The obtained shade of mix was sequentially checked with patient's ventral and dorsal aspect of finger (Fig. 8).

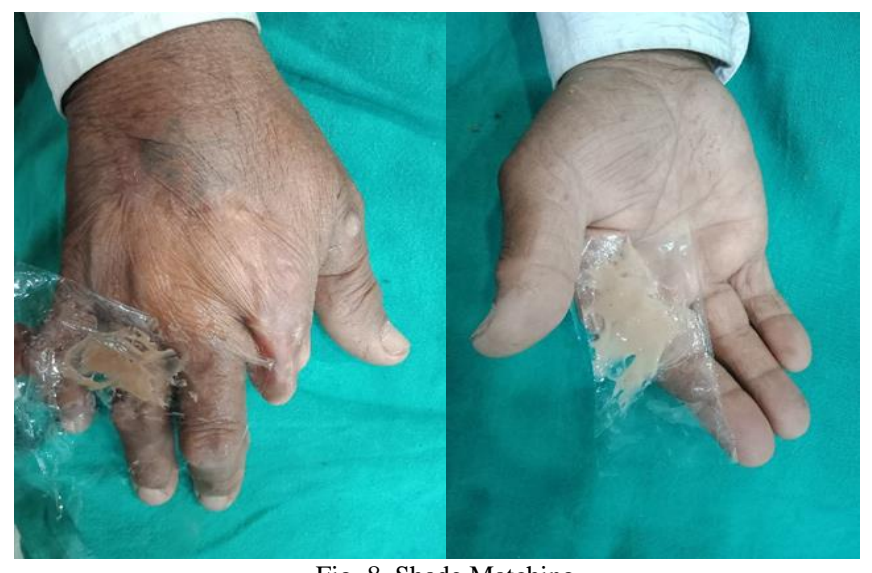

Fig. 8. Shade Matching.

\section{Flasking And Processing of Finger Prosthesis}

After a satisfactory trial, the wax pattern was flasked using ADA type III dental stone (Kal Rock, Kala Bhai Karson Pvt.,) into flexible denture curing flask using two pour technique (Fig. 9). Necessary steps were taken to avoid incorporation of air bubbles. It was steered clear of any undercuts to facilitate easy opening of the flasks and ensuing removal of the set silicone prosthesis. Dewaxing was carried out, and mold was obtained (Fig. 16).

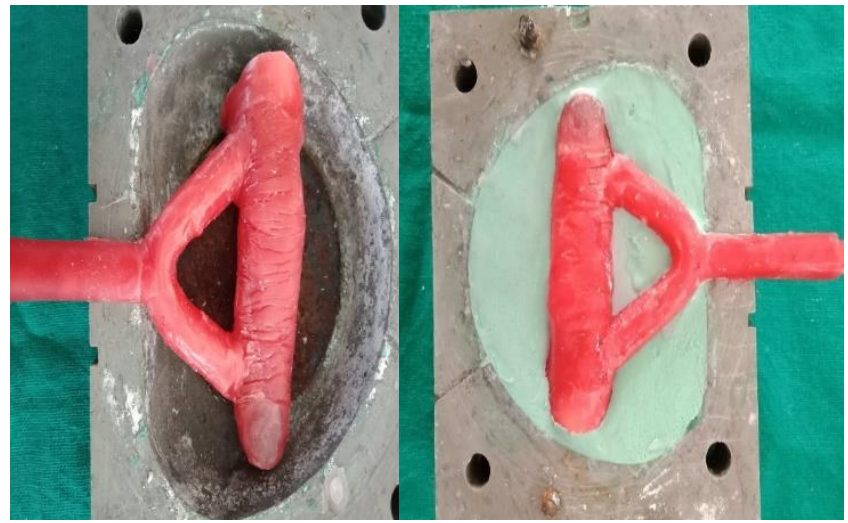

Fig. 9. Flasking.

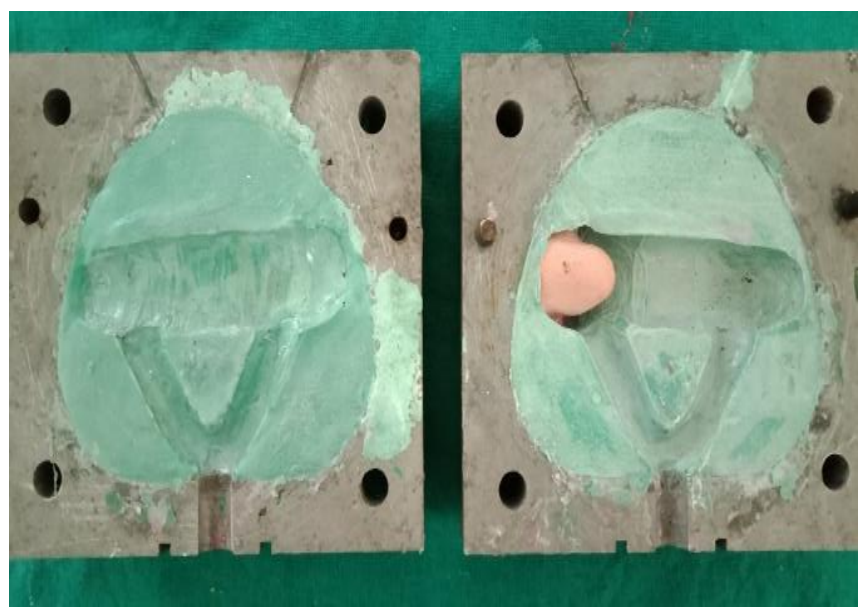

Fig. 10. Dewaxed Mould. 
Sodium alginate solution was then applied into mold and on prepared stump as a separating media and complete closure of flask was again checked. The mix was divided into two parts one for dorsal, and other for ventral portion of the finger. The shade of the material to be packed was matched to that recorded during trial. Once satisfactory shade was obtained, the initial layer of the material was painted on the dorsal and ventral surfaces separately with help of a brush. The material was left for $24 \mathrm{hrs}$ for initial polymerization. The remaining material was injected with the help of a syringe after the initial polymerization had occurred. The flasks were cured for $1 \mathrm{hr}$ at $180^{\circ} \mathrm{C}$. Deflasking was done, and prosthesis was carefully retrieved from stump. Any extra material was trimmed by means of sharp curved pair of scissors (Fig. 11, 12). The prosthesis was finished with the help of burs supplied by the company. The fit, form and colour of the prosthesis were evaluated on the patient (Fig 13-15).

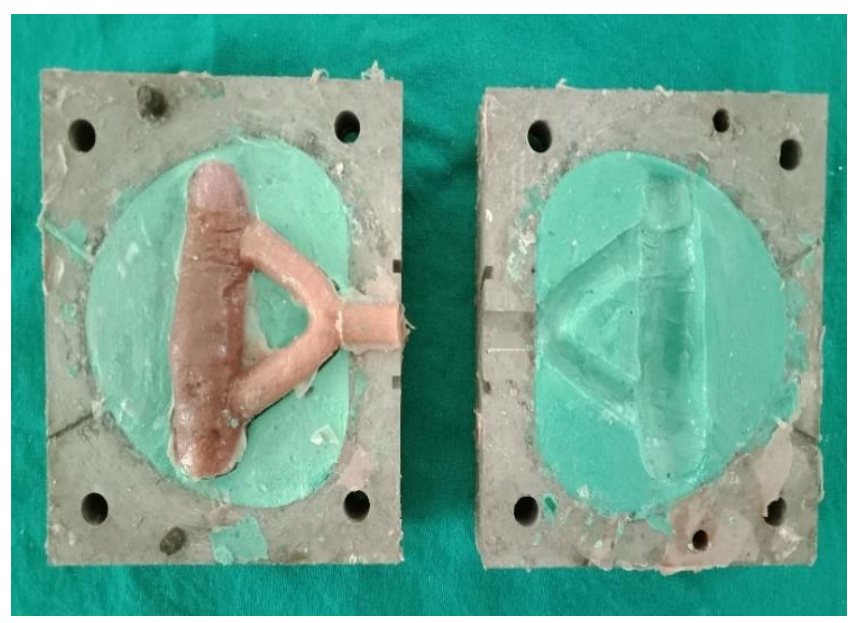

Fig. 11. Processed Prosthesis.

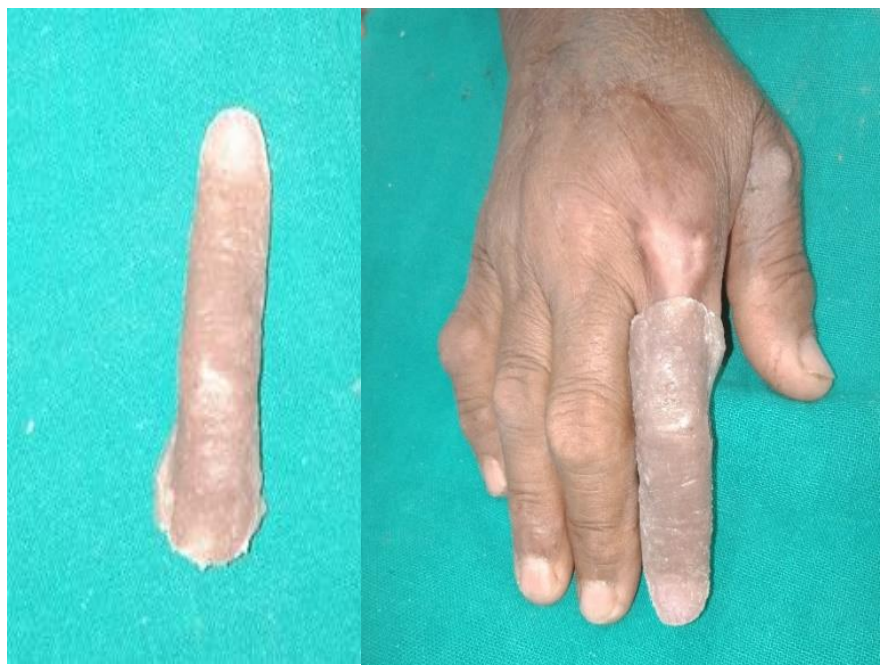

Fig. 12. Finished Prosthesis.

Fig. 13. Final Prosthesis.

A special adhesive (Suvidha Eye Services, New Delhi) was given to the patient to apply on the intaglio surface of the prosthesis to aid in its retention. A customized ring was fabricated to conceal the joint of the prosthesis and the remaining stump, and to provide extra retention. The patient was given instructions regarding maintenance of the prosthesis. Special instructions were given to avoid moisture contact and direct sunrays exposure. Patient was recalled after 1 week for checkup.

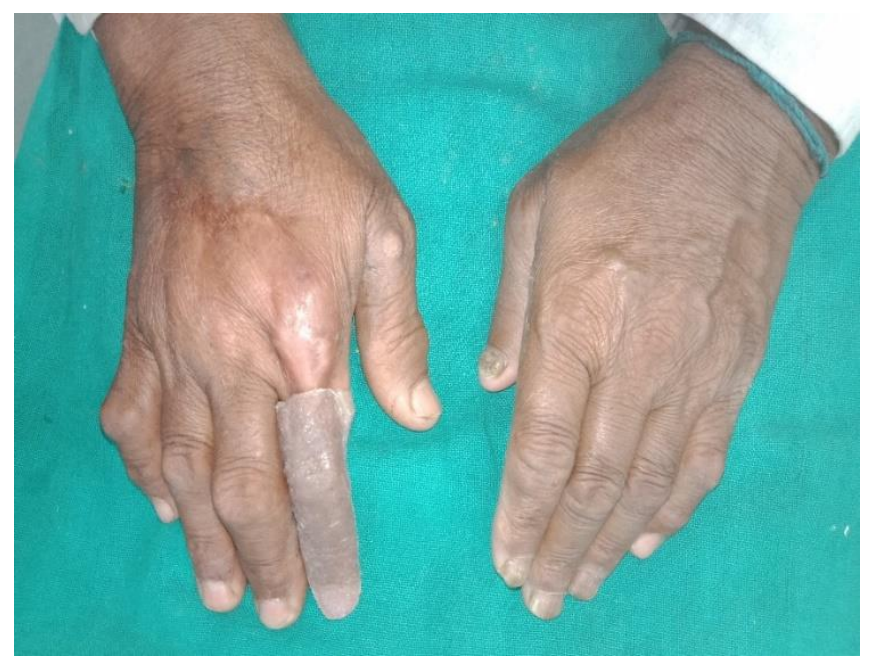

Fig. 14. Final Prosthesis.

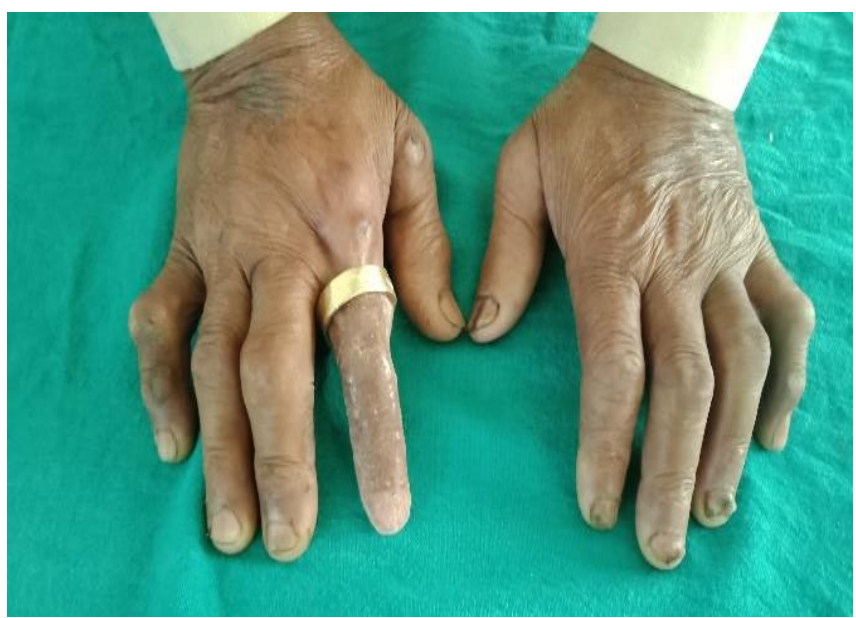

Fig. 15. Final Prosthesis with Ring for Auxiliary Retention.

\section{DISCUSSION}

Loss of limb or even a part of it affects the esthetics and function, massively impacting the dexterity of a person. Most cases involving phalange amputations can be restored to near normal functionality using appropriate prosthesis. The restoration of such defects can be done by either delivering a functional or non-functional prosthesis. Functional prosthesis delivered have an advantage of carrying out some limited movements such as holding a piece of paper or bottle. But its disadvantage lies in the fact that these prostheses are quite heavy with many electrodes placed on the forearm which do not resemble like a normal finger at all. Also, these are quite expensive and cannot be afforded by the masses.

Therefore, despite the advances in the techniques, number of patients are still more benefitted with a passive prosthesis. So, a passive prosthesis with different retentive methods is still the choice most commonly opted for. The primary purpose of this 
type of prosthesis is to allow the patient to pass unnoticed, and concealment of prosthesis usage has been found to be an effective coping strategy [7].

The traditional method of prosthesis is replacing the lost finger by an artificial digit. Current material of choice being silicone elastomers (chemical name-polysiloxane). The finger prosthesis requires an optimum retention for functions such as grasping, carrying, and holding. A significant advantage of using this technique is the exact duplication of the anatomical and the fine surface details of the digits. This allows the surface characteristics of the prosthesis to be closely matched to that of the remaining digits of the hand. With the appropriate alignment modifications, the technique can be applied to cases of partial hand amputations involving the other digits, including the thumb. Owing to the wider rate of acceptance, comfort, durability, stain resistance of customized silicone prostheses and patients' unwillingness to go for any surgical procedure involving implants, the above-explained method of fabrication of prosthesis was opted. The additional functional benefits of silicone prosthesis include desensitization and protection of the painful hypersensitive tissue at the amputation site by constant gentle pressure exerted over the affected area. It has also been speculated that silicone gel improves the hydration comfortable. The type of retention opted for depends upon the length of the stump, nature and resiliency of the underlying tissue, presence of bony undercut or subcutaneous nodules. As the stump in this case was sufficient in length to support a prosthesis and the overlying tissue was resilient enough, the traditional glove type method of retention was used. Various methods of retention are available such using rings over the margins of the finger prosthesis, using medical grade adhesive, or by implant-retained prosthesis [81]-[10] making its use vaster and more comfortable.

\section{CONCLUSION}

An acceptable prosthesis is a mixture of good suspension and camouflaging which makes it acceptable and thus routinely used by the patient. In addition to these patient's likeliness, comfort and cost factors should also be taken into consideration.

Characteristics such as accurate shape, thin margins, life-like appearance with realistic color and detailed characterization such as fingernail, contours and skin folds are also important for patient satisfaction.
This level of restoration is more successful when finger prosthesis is individually sculpted and colored in-situ under a variety of lighting conditions.

\section{CONFLICTS OF INTEREST}

There are no conflicts of interest.

\section{REFERENCES}

[1] Yeo CJ, Sebastin SJ, Chong AK. Fingertip injuries. Singapore Med J2010; 51:78-86.

[2] Shanmuganathan N, Uma Maheswari M, Anandkumar V,Padmanabhan TV, Swarup S, Jibran AH. Aesthetic finger prosthesis. JIndian Prosthodont Soc 2011; 11:232-7.

[3] The Glossary of Prosthodontic Terms: Ninth Edition. J Prosthet Dent. 2017 May;117(5S):e1-e105. doi: 10.1016/j.prosdent.2016.12.001.

[4] Kaira SL, Handa M, Dabral E and Gupta KK. Silicon Finger Prosthesis for A Partial Finger Amputation: A Case Report. AJPP 2014 105-111.

[5] Buckner H. Cosmetic hand prosthesis a case report. J ProsthetOrthot 1980; 3:41-5.

[6] Kini AY, Byakod PP, Angadi GS, Pai U, Bhandari AJ. Comprehensive prosthetic rehabilitation of a patient with partial finger amputations using silicone biomaterial: A technical note. ProsthetOrthot Int2010; 34:488-94.

[7] Yeshwante B, Parasrampuria N, Baig N. Prosthetic rehabilitation of anamputated finger. IOSR J Dent Med Sci 2014; 13:10-7.

[8] Larcher S, Espen D. Post-acute management of fractures of the proximal interphalangeal joint with metal prosthesis: first experience. HandchirMikrochirPlastChir2007; 39:263-6.

[9] Onishi Y, Fujioka H, Doita M. Treatment of chronic post-traumatic hyperextension deformity of proximal interphalangeal joint using the suture anchor: A case report. Hand Surg 2007; 12:47-9.

[10] Bickel KD. The dorsal approach to silicone implant arthroplasty of the proximal interphalangeal joint. J Hand Surg Am 2007; 32:909-13.

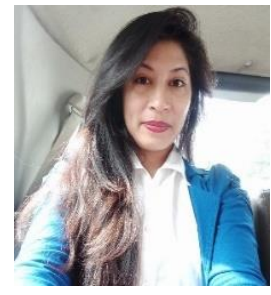

Simarpreet Kaur was born in Punjab 1993. Dr. Simarpreet Kaur graduated from National Dental College and Hospital in 2017 and is pursuing Masters (final year) in Prosthodontics and Crown \& Bridge from Baba Farid University of Health Sciences, Punjab.

Among the publications:

Kaur, M., Kaura, S. M., Sharma, A., \& Kaur, S. (2021). Interim Prosthesis for Repositioning of Mandible in a Hemimandibulectomy Patient: A Case Report. European Journal of Medical and Health Science, 3(2), 42-45. https://doi.org/10.24018/ejmed.2021.3.2.801. 